\title{
The Effectiveness of Gefitinib on Spinal Metastases of Lung Cancer - Report of Two Cases -
}

\author{
Mineyuki Zukawa*, Masato Nakano ${ }^{\dagger}$, Norikazu Hirano ${ }^{\dagger}$, \\ Keiichi Mizuhashi ${ }^{\dagger}$, Masahiko Kanamori* \\ *Department of Orthopedic Surgery, University of Toyama, Faculty of Medicine, Toyama, Japan, \\ ${ }^{\dagger}$ Department of Orthopedic Surgery and Center for Respiratory diseases, Toyama Rosai Hospital, Toyama, Japan
}

Lung cancer has a high mortality rate and is often diagnosed at the metastatic stage. Recently, gefitinib, a molecule target therapeutic drug, has offered a new approach for patients with non-small-cell lung cancer (NSCLC). This report describes the effects of gefitinib on bone metastases in two patients with NSCLC. The pain induced by a bone metastasis was relieved after the administration of gefitinib. Furthermore, the radiographs and CT findings showed sclerotic changes that matched those of the metastatic bone tumor after gefitinib administration in both patients. It is believed that gefitinib inhibited tumor cell proliferation and induced normal bone formation. In patients with NSCLC, gefitinib may be effective in the treatment of bone metastases.

Key Words: Gefitinib, Bone metastases, Lung cancer

\section{Introduction}

The prognosis of lung cancer patients with bone metastases is very poor due to the lack of an effective treatment. Recently, "gefitinib" (Iressa; AstraZeneca, Wilmington, DE, USA), a new therapeutic drug for non-small-cell lung cancer, has become available for clinical use. Inhibitors of epidermal growth factor receptor (EGFR) tyrosine-kinase are a new therapeutic option for non-small-cell-lung cancer (NSCLC) patients in-locally advanced or metastatic stages after being treated with platinum-based chemotherapy ${ }^{1,2}$. This paper presents two cases showing the remarkable effectiveness of gefitinib on bone metastases of a lung adenocarcinoma. The potential therapeutic effects of gefitinib on bone metastases are discussed with a review of the relevant literature

\section{Case Reports}

\section{Case 1}

A 52-year-old man presented with gradually increasing pain in his neck and left upper arm over a 3 month period. When he had visited our hospital, he could not move his neck as a result of the pain. The plain radiographs demonstrated a collapsed vertebral body at C-6 (Fig. 1A). The CT scan findings of the lung revealed a solitary round tumor with an irregular margin in the left upper lobe. The size was $2 \mathrm{~cm}$ in diameter. Technetium-99 bone scintigraphy demonstrated multiple spinal bone metastases, which were diagnosed as a stage IV (T1NOM1).

The patient underwent posterior decompression and spinal fusion as palliative surgery. The visual analog scale (VAS) improved to $2 \mathrm{~cm}$ from $10 \mathrm{~cm}$. The pathological diagnosis was adenocarcinoma. Although platinum-based

Received Sep 15, 2008; 1st revised Oct 13, 2008; accepted Oct 15, 2008

Corresponding author: Mineyuki Zukawa, MD

Department of Orthopedic Surgery, University of Toyama, Faculty of Medicine

Sugitani2630, Toyama 930-0194, Japan

Tel: +81-76-434-7353, Fax: +81-76-434-5035, E-mail: zukawa@med.u-toyama.ac.jp 
chemotherapy was administered, the tumor marker and size did not improve. The patient rejected additional therapy. Four months after the first operation, the C3-6 vertebral body collapsed and he could sit up (Fig. 1B). Therefore, the patient underwent $\mathrm{C} 2-7$ anterior body fusion as palliative surgery again. After surgery, the neck pain was reduced, and the VAS was improved to $4 \mathrm{~cm}$ from $10 \mathrm{~cm}$. However, two months after the second operation, he presented with intolerable neck pain. The performance status deteriorated to 3. Since the radiograph showed the progress of osteolysis (Fig. 1C) and the primary focus progressed, the oral administration of gefitinib $250 \mathrm{mg} /$ day was started as a second-line chemotherapy with informed consent. After administration for one month, the pain was reduced to allow walking. The performance status improved to ${ }^{2}$. The VAS improved to $4 \mathrm{~cm}$ from $10 \mathrm{~cm}$. At three months after administration, the cervical metastatic lesions showed remarkable increasing bone formation (Fig. 1D). The CT scan findings of the cervical vertebrae confirmed osteosclerosis matched with the metastatic bone tumor lesion (Fig. 2). The patient survived for a further two years after having a recognized bone metastasis. The activity of daily living (ADL) continued during the term of gefitinib administration.
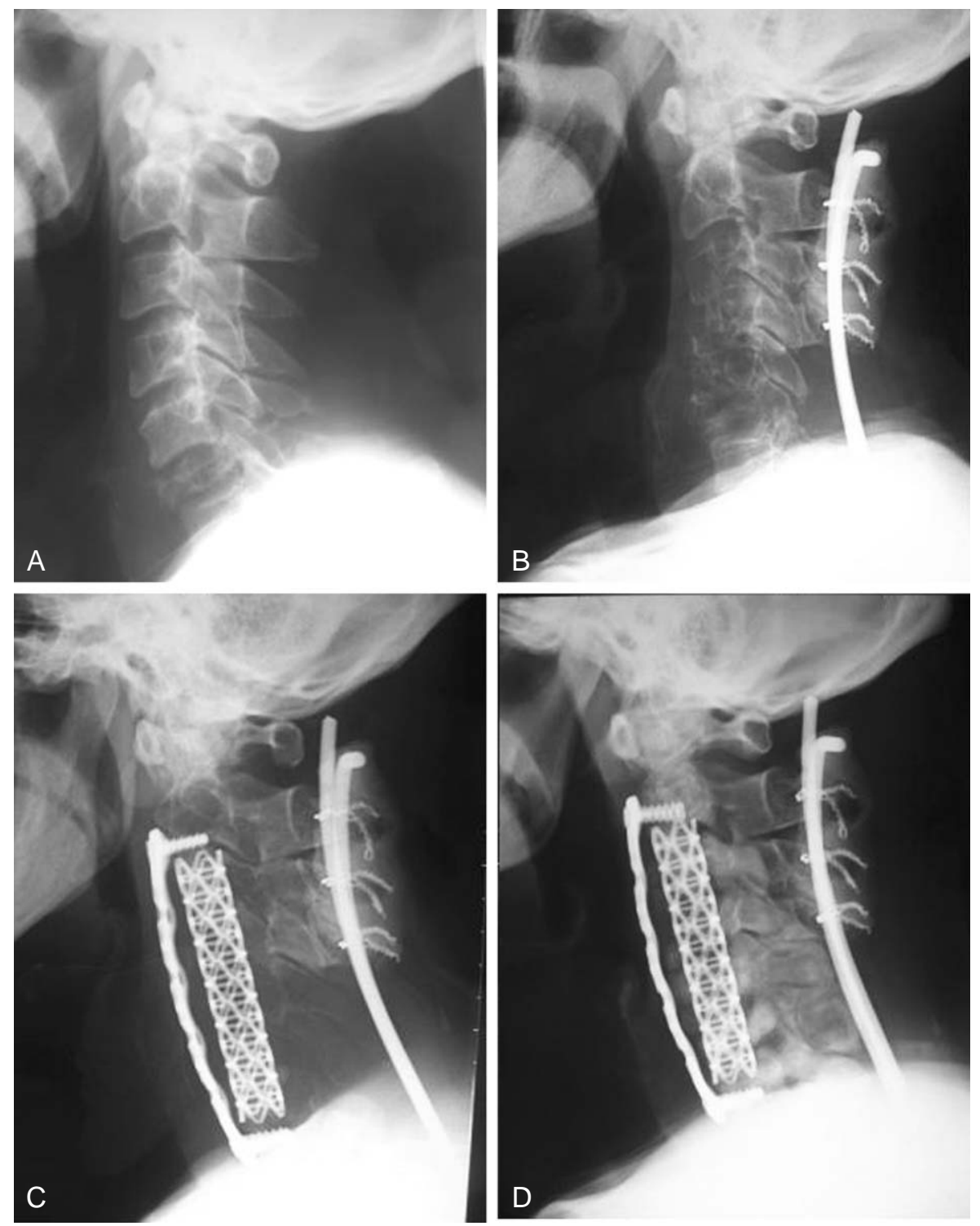

Fig. 1. Case 1. Plain radiographs of the cervical spine (A) Prior to surgery; radiograph showed collapse of the vertebral body at C-6 (B) four months after the first operation; C3-6 vertebral body collapse advanced (C) two months after the second operation (before gefitinib administration); radiograph showed the progress of osteolysis. (D) 3 months after gefitinib administration; cervical metastatic osteolytic lesions show remarkablly increasing osteosclerosis. 


\section{Case 2}

A 78-year-old woman presented with cough and weight loss over a 3 month period. The plain radiographs and CT scan findings revealed a tumor in the left upper lobe of the lung. A pathological diagnosis by sputum suggested an ade-
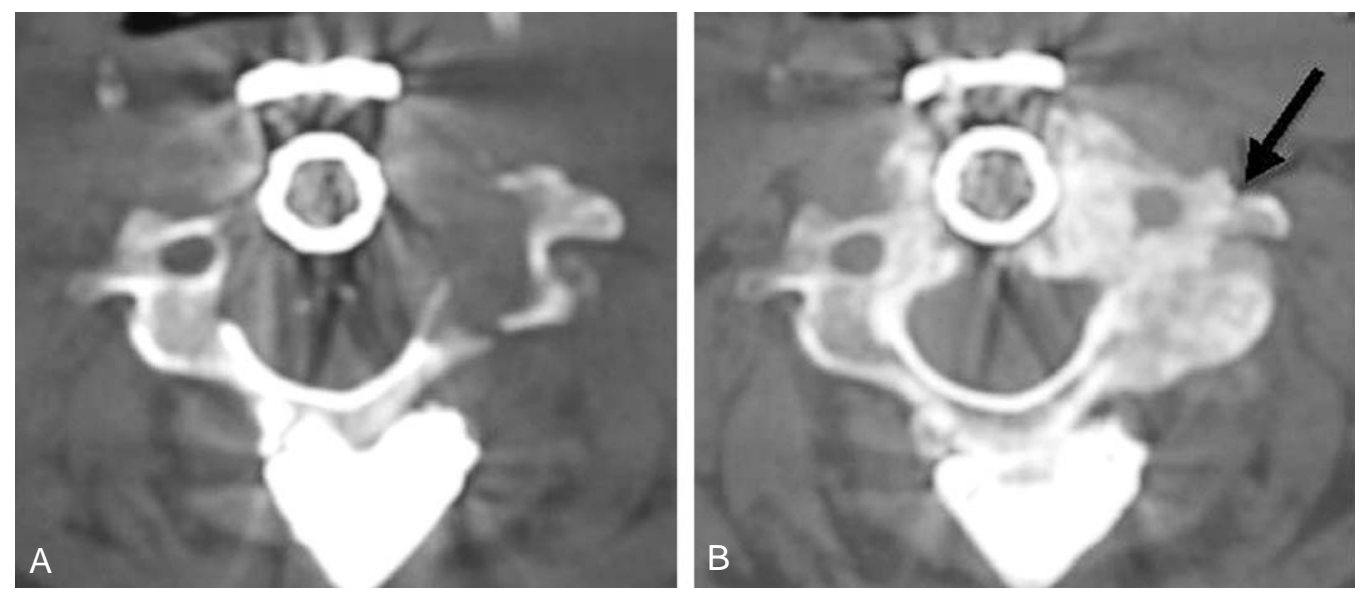

Fig. 2. CT scans showing osteosclerosis in the metastatic bone tumor area at C-5. (A) CT scans obtained immediately before gefitinib administration. (B) CT scans demonstrating remarkable bone formation at 3-months after gefitinib administration.
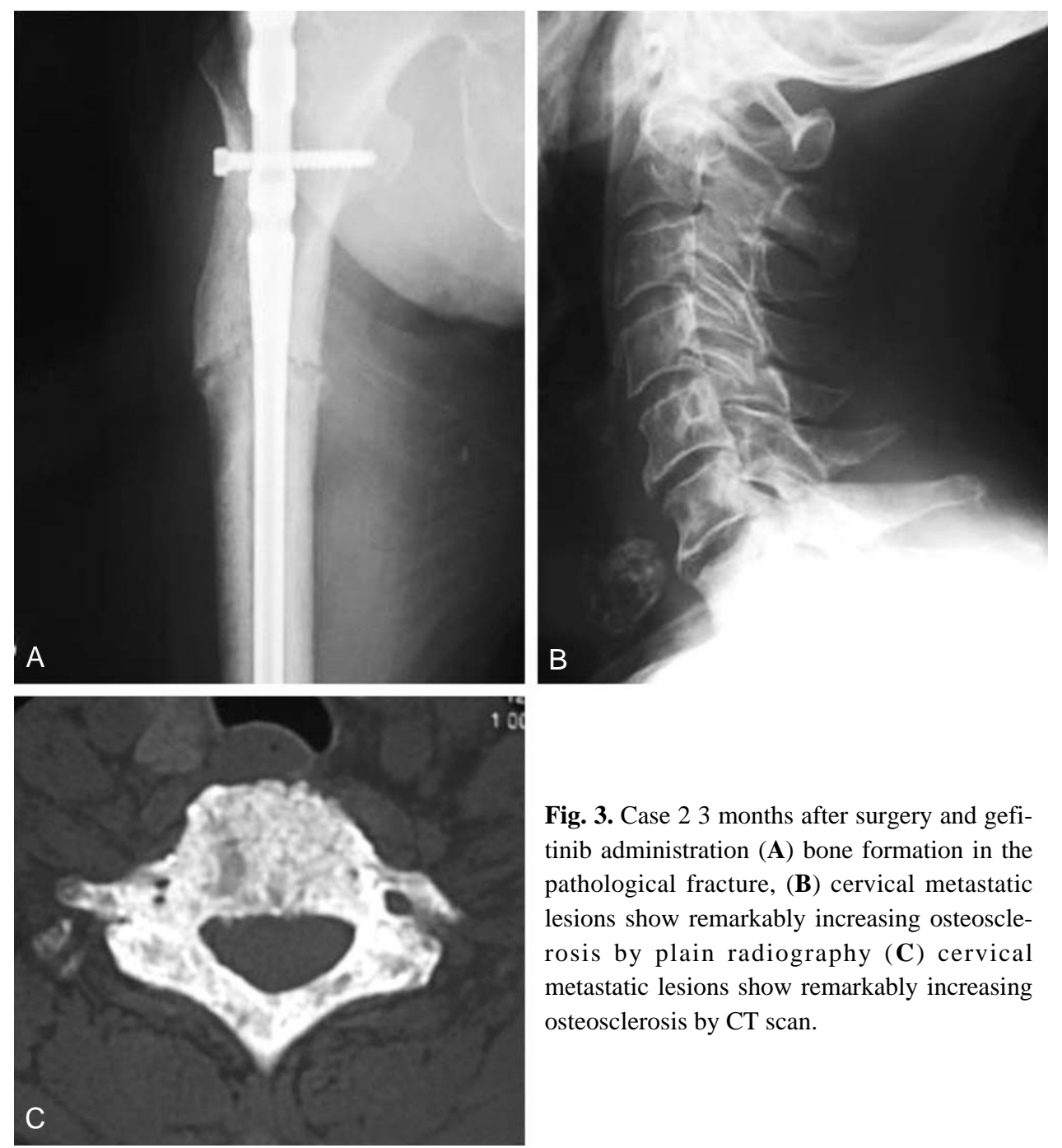

Fig. 3. Case 23 months after surgery and gefitinib administration (A) bone formation in the pathological fracture, (B) cervical metastatic lesions show remarkably increasing osteosclerosis by plain radiography (C) cervical metastatic lesions show remarkably increasing osteosclerosis by CT scan. 


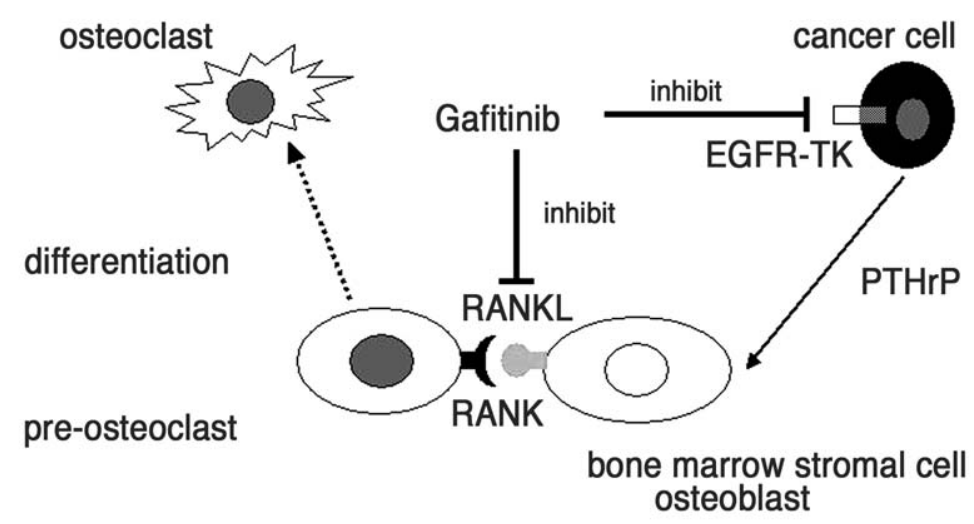

\section{(N Normanno et.al Endocrine-Related Cancer 2005)}

Fig. 4. Gefitinib inhibits osteoclast differentiation in two ways. gefitinib inhibits osteoclast differentiation and restrains bone destruction by reducing the expression of RANKL, inhibiting EGFR-TK in cancer cells and inhibiting osteoclast activation factor (PTHrP) from cancer cells.

nocarcinoma. In addition, to the brain metastases, there were multiple bone metastases of the pelvis, spine and femur. She was diagnosed with stage IV (T4N3M1) lung cancer. The oral administration of gefitinib $250 \mathrm{mg} /$ day was commenced with informed consent. One week later, she suffered from the pathological fracture of the femur, and underwent internal fixation. Three months after surgery, remarkable bone formation and union was observed in the plain radiograph (Fig. 3A). She was able to walk without pain. The cervical metastatic lesions revealed remarkable bone formation in the plain radiographs and CT scan findings (Fig. 3B and C). She was still alive one year after the recognized bone metastasis with an improvement in the pain and ADL after gefitinib administration.

\section{Discussion}

Lung cancer has a high mortality rate and is often diagnosed at the metastatic stage. Recently, gefitinib has offered a new approach for patients with NSCLC. Response rates of between 9 18\% have been reported in phase II trials for gefitinib in unselected patients with NSCLC. A never smoking status, female gender and Asian ethnicity are associated with an increased sensitivity to gefitinib².

In our cases, the patients with bone metastases of a lung adenocarcinoma improved temporarily after palliative surgery but deteriorated a short time later. According to the prognostic score for a metastatic spinal tumor, both cases had a predicted prognosis (mortality) of within six months, and were indicated as palliative surgery, or supportive care only ${ }^{3,4}$. However, the administration of gefitinib allowed a longer period of survival and improved pain and ADL.

The plain radiographs and CT scan findings suggested remarkable bone formation matched with the metastatic osteolytic lesion. Normanno et al. ${ }^{5,6}$ reported that gefitinib inhibited the ability of human bone marrow stromal cells to induce osteoclast differentiation by reducing the level of RANKL expression. In the clinical cases, it is believed that gefitinib restrains bone destruction by inhibiting osteoclast differentiation in addition to the normal pharmacological phenomenon, where it inhibits EGFR-TK of cancer cells directly as well as the osteoclast activation factor (PTHrP) from cancer cells (Fig. 4). In hindsight, it would have been good to examine the bone growth in more detail (bone mineral density, bone turnover marker, and more). However, the patients have since died. This will certainly be examined in future patients.

To date, some studies reported that gefitinib reduces the bone or brain metastasis of NSCLS ${ }^{7}$. Their patients were treated with gefitinib and combination with bisphosphonate, radiation, etc 8 . In the present two cases, gefitinib extended their prognosis. In future, gefitinib should be considered among all current treatment options for cases where there is a surgical indication of bone metastases of lung cancer. 


\section{REFERENCES}

1. Edelman MJ: An update on the role of epidermal growth factor receptor inhibitors in non-small cell lung cancer. Semin Oncol 2005; 32: S3-S8.

2. Lange T, Muller-Tidow $C$, Serve H, Hoffknecht $P$, Berdel WE, Thomas M: First-line systemic treatment with gefitinib in stage IV non-small cell lung cancer. Oncol Rep 2005; 14: 1539-1542.

3. Tokuhashi Y, Matsuzaki H, Toriyama S, Kawano H, Ohsaka S: Scoring system for the preoperative evaluation of metastatic spine tumor prognosis. Spine 1990; 15: 11101113.

4. Tomita K, Kawahara N, Kobayashi T, Yoshida A, Murakami H, Akamaru T: Surgical strategy for spinal metastases. Spine 2001; 26: 298-306.

5. Normanno N, De Luca A, Aldinucci D, et al: Gefitinib inhibits the ability of human bone marrow stromal cells to induce osteoclast differentiation: implications for the pathogenesis and treatment of bone metastasis. Endocr Relat Cancer 2005; 12: 471-482.

6. Normanno N, Gullick WJ: Epidermal growth factor receptor tyrosine kinase inhibitors and bone metastases: different mechanisms of action for a novel therapeutic application? Endocr Relat Cancer 2006; 13: 3-6.

7. Wu C, Li YL, Wang ZM, Li Z, Zhang TX, Wei Z: Gefitinib as palliative therapy for lung adenocarcinoma metastatic to the brain. Lung Cancer 2007; 57: 359-364.

8. Katakami N: Lung cancer with bone metastasis. Gan To Kagaku Ryoho 2006; 33: 1049-53. 